\title{
INSTRUCTION AND RESEARCH IN APPLIED MATHEMATICS ${ }^{1}$
}

The history of mathematics reveals a continuous interplay of ideas between the mathematician and the experimental or practical scientist. In the last half-century this interplay has decreased, to the detriment, it may be believed, of both parties. The war caused a lively cooperation, but unless this continues under peace-time conditions, the prospect for the future is serious and warrants earnest consideration.

The widening gap between the mathematician and other scientists may be traced to several causes. The most obvious of these is the great activity in all branches of science, which has necessitated a high degree of specialization in each branch, and consequently less opportunity for roaming interests. However, side by side with the expansion of science, increased understanding of basic theory acts as a simplifying compensation. Such simplification is achieved largely through mathematical methods, of increasing depth and generality, and it would be indeed unfortunate if mathematicians should not be in a position to contribute towards this simplification.

As our educational system is at present organized, there are relatively few opportunities for a student of mathematics, undergraduate or graduate, to become acquainted with the mathematical structures of the theories underlying other sciences. His needs in this connection are not identical with those of students pursuing other branches of science as their major interest; many practical details, of importance to them, are not essential for his purpose, and he can go further and faster by concentration on the mathematical aspects of the subject. It is suggested that the study of some branches of other sciences from the mathematical standpoint (and that is a meaning commonly attached to the words "applied mathematics") should be regarded, wherever feasible, as an essential part of the training of mathematics majors, undergraduate or graduate. Apart from the general advantage to science which we might hope to see as a result of this procedure, it seems only fair to offer a young mathematician the opportunity of pursuing a career in applied mathematics if his natural inclinations are so directed. Unless he has the opportunity of viewing the field of applied mathematics from the mathematical standpoint, it is unlikely that he will be attracted to it if his interests are basically those of a mathematician.

\footnotetext{
1 Prepared by the Special Committee on Applied Mathematics and approved by the Council of the American Mathematical Society on April 26, 1947.
} 
Consequently it is suggested that departments of mathematics throughout the country should consider the feasibility of enlarging their offerings in the direction of applied mathematics, both on the undergraduate and graduate levels, insofar as the departments are qualified to offer such instruction. Many branches of applied mathematics have not only well established axiomatic mathematical structures, but are also fruitful fields of research, involving mathematical ideas and techniques of the highest order. Attention may in particular be directed to mathematical statistics, theoretical mechanics (including elasticity and fluid dynamics), statistical mechanics and thermodynamics, heat conduction, electromagnetic theory, relativity, quantum mechanics, genetics, and the theory of high polymers.

In some institutions the problem is already solved in part by the activities of other departments, containing members well qualified in mathematics. Never theless, since each of the subjects listed above has not only its mathematical side, but also an even greater experimental or practical side, it appears likely that full justice can be done to the mathematical theories only by a specialization on that aspect. Needless to say, the task of maintaining and increasing an interplay between mathematics and the other sciences is one that can be dealt with only by full cooperation between all the parties concerned. It is to be expected that scientists in other fields would welcome the desire of young mathematicians to increase their knowledge of the mathematical structures of those fields, for the sake of a wider mathematical perspective and, in the case of those qualified to pursue research, with a view to solving outstanding problems and simplifying basic theory by mathematical methods of increased generality and power.

A similar gap in the background of our students lies in the field of computation. Even if we carefully distinguish mathematical computation and computation engineering, there is much of importance concerning numerical computation and its organization for hand computer, IBM equipment, relay calculator or electronic computer, which will be of value to the student whether he is to become a topologist, a flutter analyst, or a mathematical logician. Of equal importance is the field of algebraic and analytic computation and its possibilities of mechanization. With the exception of a few institutions where the engineers have begun to work on computation engineering, this whole field is at the disposal of the departments of mathematics.

Consequently, it is suggested that departments of mathematics throughout the country should consider the feasibility of enlarging their offerings in computation, algebraic, analytic, or numerical, at both the undergraduate and graduate levels. 